\title{
AVALIAÇÃO DA PERCEPÇÃO DO ENVELHECIMENTO VOCAL EM IDOSOS
}

\author{
Mauriceia Cassol
}

\section{Resumo}

Este estudo objetiva avaliar a voz de um grupo de idosos relacionando a qualidade vocal e seu grau de alteração com o impacto causado em relação à vida particular, profissional e social, através das avaliações perceptivoauditiva e da utilização de uma escala de auto-avaliação vocal. Fizeram parte deste estudo 19 indivíduos idosos do gênero masculino e gênero feminino na faixa etária de 60 a 80 anos, alunos da Universidade do Adulto Maior do Centro Universitário Metodista IPA. Para a análise perceptivo-auditiva foi realizada a gravação digital da voz através da emissão da vogal /a/ sustentada e da contagem de números de um a 20. Na realização da auto-avaliação da psicodinâmica vocal os indivíduos foram instruídos à marcar numa escala de zero a dez o impacto da sua voz em relação à vida particular, profissional e social, sendo considerado o impacto vocal de zero a três ruim, de quatro a sete bom e de oito a dez ótimo. Na avaliação perceptivo-auditiva da qualidade vocal houve predomínio do tipo de voz rouca-soprosa de grau leve, sendo um dos principais marcadores do envelhecimento da voz ou presbifonia. $\mathrm{Na}$ escala de auto- avaliação vocal em relação a vida particular os indivíduos avaliaram sua voz com ótimo e bom impacto, na vida profissional consideraram que costumava causar impacto ótimo e na vida social impacto bom. Pode-se concluir que todos os indivíduos possuem uma auto- imagem vocal positiva, apesar de apresentarem uma qualidade vocal alterada decorrente do processo de envelhecimento vocal.

Palavras-chave: Voz. Idosos. Geriatria. Gerontologia.

\footnotetext{
* Fonoaudióloga, Doutora em Ciências da Saúde na área de Geriatria pela Pontifícia Universidade Católica do Rio Grande do Sul (PUCRS). Professora do Curso de Fonoaudiologia do Centro Universitário Metodista IPA e da Universidade do Adulto Maior (UAM). Pesquisadora integrante do Núcleo de Estudos e Pesquisas do Envelhecimento (NEPE). E-mail: m.cassol@terra.com.br
} 


\section{Introdução}

A Fonoaudiologia tem desempenhado um importante papel na Gerontologia, desenvolvendo pesquisas nas áreas de deglutição, linguagem, audição e sobretudo em relação aos estudos da voz no indivíduo idoso, os quais têm sido cada vez mais referidos na literatura (VENITES; BERTACHINI; RAMOS, 2004).

A percepção da velhice ou senescência normalmente acontece de "fora para dentro" e não é reconhecida de imediato pela própria pessoa, mas parte do outro ou de uma situação do cotidiano (BARBIERI, 2002, online). No entanto, algumas mudanças que ocorrem na senescência são mais facilmente reconhecidas pelo indivíduo, como a cor dos cabelos que branqueiam, o surgimento de rugas na pele, diminuição da agilidade nos movimentos, flacidez muscular, déficits sensoriais e a menopausa, além da diminuição da estatura em função da compressão vertebral, estreitamento dos discos e cifose, redução da capacidade respiratória vital e volume expiratório (MATSUDO; MATSUDO; BARROS NETO, 2000). Mas, e a voz? Será que as pessoas percebem que ela envelhece? O objetivo deste trabalho, foi avaliar a voz de um grupo de idosos relacionando a qualidade vocal e seu grau de alteração com a auto-avaliação do impacto vocal causado em relação à vida particular, profisssional e social.

\section{Literatura}

A voz é uma das extensões mais fortes da nossa personalidade e se aguçarmos nossos sentidos reconheceremos que esta extensão é mais profunda em sua dimensão não-verbal do que na verbal, além disso, em todas as situações de emissão podemos ter vários níveis de análise, de leitura vocal: leitura dos parâmetros físicos, psicológicos, sociais, culturais e educacionais de um determinado falante. Assim, a voz é o veículo de nossa inter-relação, de comunicação, um meio de atingir o outro. Qualidade vocal é o termo atualmente empregado para designar o conjunto de características que identificam a voz humana, sendo considerada o índice mais completo dos atributos da emissão de um indivíduo, capaz de nos fornecer desde informações sobre suas características físicas até dados de sua formação educacional (BEHLAU; ZIEMER, 1988). 
A voz conta com uma série de dados inerentes a três dimensões do indivíduo: a biológica, psicológica e socioeducacional. As informações contidas na informação biológica dizem respeito aos nossos dados físicos básicos tais como o sexo, idade e condições gerais da saúde; as informações contidas na dimensão psicológica correspondem às características básicas da personalidade e do estado emocional do indivíduo durante o momento da emissão; já a dimensão socioeducacional oferece dados sobre os grupos a que pertencemos, quer sejam sociais ou profissionais (BEHLAU; PONTES, 1999). Na literatura autores referem que é importante solicitar ao paciente que avalie o impacto da alteração vocal em sua vida particular, profissional e social. Geralmente, uma voz auditivamente pouco alterada, porém, com muito esforço à produção, recebe uma avaliação de impacto maior do que uma voz mais alterada, contudo sem esforço associado (BEHLAU et al., 2001).

Deve-se descrever a impressão transmitida pela voz usada, a sua possibilidade de rejeição ou aceitação social. Esse conceito está relacionado ao de audição criativa a partir de observações sobre os diferentes tipos de vozes e seus impactos (MOSES, 1954).

A avaliação da psicodinâmica vocal é a descrição do impacto psicológico produzido pela qualidade vocal do indivíduo, considerando-se desde os aspectos fonatórios propriamente ditos, até os elementos de velocidade e ritmo de fala. A relação entre aspectos de personalidade, sentimentos, emoções e voz é estudada e descrita desde o tempo dos gregos, com bastante interesse nos dias de hoje (BEHLAU; MADAZIO; PONTES, 1999).

A avaliação perceptivo-auditiva é uma avaliação clássica da qualidade vocal e tem como principal objetivo definir características vocais que são: rouca, áspera, soprosa, astênica, tensa, pastosa, trêmula, sussurrada, fluída, bitonal, diplofônica, monótona, infantilizada, presbifônica, hipernasal e hiponasal (BARROS; CARRARA-ANGELIS, 2002).

Recebe o nome de presbilaringe o envelhecimento laríngeo inerente à idade, gerando o envelhecimento vocal que, por sua vez, é chamado de presbifonia (FERREIRA; ANUNCIATTO, 2003). Na presbifonia, a qualidade vocal é influenciada por muitas variáveis: condições laríngeas (BEHLAU, 1999; ANDREWS, 1995), auditivas (FERREIRA; ANUNCIATTO, 2003; ANDREWS, 1995; DEDIVITIS; BARROS, 2002) e sociais (FERREIRA; ANUNCIATTO, 2003; ANDREWS, 1995; SATALOFF et al., 1997). O processo de deterioração da voz difere de 
indivíduo para indivíduo, considerando-se os aspectos biopsicossociais e sua biografia, ou seja, a sua história desde a fecundação até o momento atual. (FERREIRA; ANUNCIATTO, 2003; MANSUR; RADANOVIC, 2004).

\section{Material e Método}

Esta pesquisa foi desenvolvida no Centro Universitário Metodista IPA, estando inserida na UAM, a qual realiza atividades para um envelhecimento saudável, produtivo e bem-sucedido. Os idosos faziam parte do projeto "Programa de Reabilitação Vocal e Auditiva em Indivíduos Idosos." Os critérios adotados como fatores de exclusão da amostra foram: participante com infecção respiratória aguda no dia da avaliação, doença neurológica prévia (Acidente Vascular Cerebral - AVC, Parkinson, Demência), indivíduos com cirurgia de cabeça e pescoço prévia e todos aqueles que não concordaram em participar da pesquisa.

Os indivíduos idosos que fizeram parte desta pesquisa exerceram durante sua vida laboral atividades profissionais variadas, as quais tiveram diferentes demandas de uso da voz, como professores, comerciantes, vendedores, funcionários públicos, secretários, enfermeiros, dona de casa, advogados, sem nunca terem participado de treinamento específico para a voz. O grupo avaliado constou de 19 indivíduos idosos do gênero masculino e gênero feminino na faixa etária de 60 a 80 anos. Na amostra houve predomínio de indivíduos de cor branca e do gênero feminino. Todos os sujeitos são alunos da UAM do Centro Universitário Metodista IPA e freqüentam as oficinas de fonoaudiologia em encontros semanais de uma hora de duração.

As avaliações foram realizadas no primeiro dia de participação dos idosos no grupo. Na realização da auto-avaliação da psicodinâmica vocal os sujeitos receberam uma escala e foram instruídos à marcar de zero a dez 0 impacto da sua voz em relação à vida particular, profissional e social, sendo considerado de zero a três impacto vocal ruim, de quatro a sete impacto vocal bom e de oito a dez impacto vocal ótimo. O protocolo utilizado foi uma escala adaptada de pesquisas já existentes sobre auto-avaliação vocal (BEHLAU et al., 2001). O material de fala colhido para a avaliação perceptivo-auditiva da voz foi através da emissão da vogal [a], solicitandose ao indivíduo que inspirasse e emitisse o referido som, numa única freqüência, sem variação musical ou de intensidade, ou seja, que fosse emitido de modo 
habitual, logo em seguida foi solicitado a realização da contagem de números de um a 20. Para a coleta da amostra os indivíduos ficaram em pé, com os braços estendidos ao longo do corpo, e o microfone foi posicionado num ângulo de $90^{\circ}$ da boca do indivíduo. Para emissão da vogal foi mantida uma distância em torno de cinco centímetros, para evitar interferências no sinal e manter uma proporção sinal - ruído elevado. Para fala encadeada foi mantida uma distância maior, de dez centímetros, para que o ruído respiratório não contaminasse a gravação (BEHLAU; MADAZIO; PONTES, 1999). Os indivíduos foram orientados a não aproximar a boca do microfone durante a gravação. Na coleta do material de voz, foi utilizado um gravador digital (Portable MiniDisc Recorder MZ-R3) da marca SONY e um microfone profissional, condensador, estéreo, omnidirecional, sensitividade $-35 \mathrm{~dB}$, da marca LE SON modelo MP66. As gravações foram realizadas em ambiente silencioso, porém sem isolamento acústico e não foram medidos os níveis de ruído da sala. As vozes foram avaliadas e analisadas com o auxílio de duas fonoaudiólogas professoras do curso de fonoaudiologia do Centro Universitário Metodista IPA e uma aluna bolsista de iniciação científica. As examinadoras receberam um protocolo, contendo os itens à serem avaliados. As vozes foram apresentadas aleatoriamente e o julgamento de cada voz foi realizado de forma individual. O projeto do presente estudo foi aprovado pelo Comitê de Ética em Pesquisa do Centro Univesitário Metodista IPA com protocolo de registro $\mathrm{n}^{\mathrm{o}} 1076$. A pesquisadora comprometeu-se a manter confidencialidade e sigilo em relação aos dados coletados, vinculados aos nomes dos participantes da pesquisa. Após esclarecidos quanto aos objetivos e metodologia do trabalho, os idosos participantes da pesquisa assinaram um termo de consentimento informado, o qual ressalta a não existência de desconfortos ou riscos aos mesmos, e descreve os procedimentos empregados na pesquisa (GOLDIM; FRANCISCONI; CLOTET, 2000).

\section{Resultados e Discussão}

Os dados coletados foram submetidos à análise estatística, descritiva e referencial através do cálculo da média obtendo um percentual dos testes aplicados.

Partindo de uma amostra de 19 vozes de indivíduos idosos foi realizada a análise perceptivo-auditiva e os tipos de vozes encontrados estão descritos na tabela 1 (Anexo). Nos resultados encontrados 10,5\%(dois) dos indivíduos 
idosos testados não apresentaram alteração vocal, sendo este tipo de voz classificada como voz adaptada ou normal. O conceito de voz normal e voz alterada veio se modificando ao longo do tempo, sendo amplamente influenciado pelo meio a que se pertence e pela cultura em que se vive. A voz deve ser produzida pelo falante de modo adaptado, sem esforço adicional e com conforto, identificando o sexo e a faixa etária a que pertence, e deve ser adaptada ao grupo social profissional e cultural do indivíduo (PONTES et al., 2002). O tipo de voz classificado como rouco-soprosa em grau leve foi encontrado em $47 \%$ (nove) dos indivíduos, havendo um predomínio deste tipo de voz na população testada, $16 \%$ (três) dos indivíduos apresentaram tipo de voz rouco-soprosa em grau moderado, $10,5 \%$ (dois) dos indivíduos apresentaram voz do tipo soprosa de grau leve. Foi encontrado em apenas $5,3 \%$ (um) dos indivíduos voz do tipo rouca e áspera em grau leve e soprosa em grau moderado. A voz que combina os tipos rouca, áspera, soprosa e trêmula em grau moderado foi encontrada em 5,3\% (um) dos indivíduos testados. Apenas 5,3\% (um) da amostra apresentou os tipos de voz roucosoprosa - áspera de grau leve. A voz rouca possui característica ruidosa, com altura e intensidade freqüentemente diminuídas, enquanto na voz áspera o som provoca uma sensação desagradável e até mesmo irritante. A voz áspera também é definida como uma impressão psicoacústica da irregularidade de vibração das pregas vocais, isto é, corresponde a flutuações irregulares na freqüência fundamental e/ou na amplitude da fonte sonora glótica (BEHLAU; AZEVEDO; PONTES, 1999; BEHLAU; PONTES, 1995). Na qualidade vocal soprosa ouvimos a voz acompanhada de ar não-sonorizado pelas pregas vocais; assim, temos a presença audível de um ruído à fonação, que é o fluxo contínuo de ar através da glote. A voz soprosa típica é de intensidade baixa e altura grave; porém, por um esforço de compensação para tentar reduzir o escape de ar, podemos encontrar essa qualidade vocal com intensidade forte (BEHLAU; PONTES, 1995).

No teste de auto-avaliação vocal (Tabela 2, Anexo) foram obtidos os seguintes resultados: quando perguntados sobre o impacto da voz em sua vida particular, $42 \%$ (oito) dos indivíduos testados consideraram que a voz têm um impacto ótimo, $47 \%$ (nove) consideraram impacto vocal bom e apenas $10 \%$ (dois) consideraram impacto vocal ruim. Em relação ao impacto vocal durante a vida profissional, $53 \%$ (dez) dos indivíduos consideraram ótimo, $36 \%$ (sete) consideraram impacto vocal bom e apenas 10\% (dois) impacto vocal ruim. Em relação ao impacto vocal na vida social, 31\% (seis) 
consideraram impacto vocal ótimo, 57\% (11) impacto vocal bom e apenas $10 \%$ (dois) impacto vocal ruim. Nesta pesquisa ocorreram divergências entre as avaliações em relação à impressão transmitida pelo tipo de voz e sua alteração, pois grande parte dos indivíduos idosos que apresentava características de voz presbifônica não percebeu essa alteração. A voz presbifônica é uma voz com grau variado de deterioração, que se expressa na falta de sustentação de freqüência, intensidade e qualidade de emissão, sendo constantes as quebras de sonoridade. É observada nos indivíduos idosos, com alterações mais evidentes após os 65 anos de idade, e mais acentuadas no sexo masculino (BEHLAU; AZEVEDO; PONTES, 1999). Ao relacionarmos a qualidade vocal e seu grau de alteração com a autoavaliação vocal, foi possível verificar que nos sujeitos com voz adaptada ou normal houve um predomínio do impacto vocal ótimo na vida particular, profissional e social. No tipo de voz rouco-soprosa de grau leve os indivíduos consideram impacto vocal bom na vida particular, profissional e social. $\mathrm{O}$ indivíduo que apresentou tipo de voz rouco-soprosa-áspera de grau leve, também referiu impacto vocal bom na vida particular, profissional e social. $\mathrm{O}$ indivíduo avaliado com tipo de voz rouca e áspera de grau leve e soprosa em grau moderado considerou impacto bom para a vida particular e social e ótimo para a vida profissional, sendo encontrado o mesmo resultado nos indivíduos com tipo de voz rouco-soprosa de grau moderado. Os indivíduos que apresentaram voz do tipo soprosa em grau leve, classificaram o impacto de suas vozes como ótimo em relação a vida particular, profissional e social. O indivíduo que apresentou a voz com as combinações rouco-áspera-soprosatrêmula de grau moderado referiu que a voz possui um impacto ótimo na vida particular, profissional e social.

No final da gravação a maioria quis ouvir a própria voz e ao se ouvirem, vários se surpreenderam dizendo que aquela voz era feia e parecia de uma pessoa muito idosa. Poder-se-ia relacionar a falta de percepção do envelhecimento da própria voz pela falta de experiências vocais dos indivíduos e pelo fato dos testes terem sido realizados no primeiro dia de participação no grupo de fonoaudiologia, ou ainda porque quando falamos, a nossa voz é percebida por via óssea e via aérea e ao escutarmos a gravação, existe a percepção do som somente por via aérea. A auto-percepção vocal foi objeto de estudo, na qual de 107 mulheres, com idade entre 37 e 71 anos, que passaram por avaliação fonoaudiológica e otorrinolaringológica, 54\% não perceberam mudança em suas vozes, mas àquelas que perceberam associaram 
à menopausa (SCHNEIDER et al., 2004). Outra pesquisa sobre a percepção vocal na terceira idade foi realizada com uma população de 100 mulheres entre 60 e 95 anos que responderam questões de auto-percepção sobre corpo, voz e rejuvenescimento vocal, e obtiveram como resultado que idosas perceberam o envelhecimento físico de forma similar, não ocorrendo o mesmo com a percepção do envelhecimento vocal; concluindo que os idosos pouco percebem o envelhecimento vocal e muitos desconhecem a possibilidade de rejuvenescer a voz (POLIDO; MARTINS; HANAYAMA, 2005).

\section{Conclusão}

Neste estudo tornou-se motivante avaliar como o indivíduo idoso percebe a sua voz. Na avaliação perceptivo-auditiva da qualidade vocal houve predomínio do tipo de voz rouca-soprosa de grau leve, sendo um dos principais marcadores do envelhecimento da voz ou presbifonia. Na avaliação da auto-imagem vocal em relação a vida particular os indivíduos avaliaram sua voz com ótimo e bom impacto, na vida profissional consideraram que costumava causar impacto ótimo e na vida social impacto bom. Observou-se ao relacionar a qualidade vocal e seu grau de alteração com o impacto vocal causado em relação à vida particular, profissional e social que os resultados obtidos foram positivos mesmo em vozes com qualidade vocal alterada, havendo um predomínio do impacto bom e ótimo na auto-avaliação vocal dos idosos. Pode-se concluir que todos os indivíduos participantes desta pesquisa possuem uma auto-imagem vocal positiva, apesar de apresentarem uma qualidade vocal alterada decorrente do processo de envelhecimento vocal. Algumas pessoas têm uma boa imagem formada sobre sua voz e sobre o impacto que ela exerce sobre o ouvinte. Outros nunca param para pensar no assunto. De qualquer forma, conscientes ou não, influenciamos com nossas vozes e somos influenciados pelas vozes das pessoas com quem fazemos contato. 


\title{
ASSESSMENT OF VOCAL AGING PERCEPTION IN THE ELDERLY
}

\begin{abstract}
This study aims to assess the voice of an elderly group relating voice quality and its alteration degree with the impact caused on professional, social and private life, through perceptive-auditory assessments and use of a voice self-evaluation scale. 19 female and male elderly individuals at ages ranging from 60 to 80 years, who were students at the IPA Adult University, participated in this study. For the perceptive-auditory analysis, a digital voice recording was performed through the emission of the sustained /a/ vowel and number counting from one to twenty. While performing the vocal psychodynamic self-evaluation, the subjects were instructed to rate from zero to ten in a scale the impact of their voices in relation to private, professional and social life, the vocal impact from zero to three being considered bad, from four to seven good, and from eight to ten optimal. In the perceptiveauditory evaluation of voice quality, there was a dominance of the hoarse, breathy voice type of mild degree, this being one of the markers of voice aging or presbyphonia. In the voice self-evalution scale in relation to private life, the subjects evaluated their voice as optimal and with good impact; regarding professional life, they considered it to cause an optimal impact; and regarding social life, a good impact. We can conclude that all subjects have a positive voice self-evaluation, despite presenting an altered voice quality stemming from the vocal aging process.

Keywords: Voice. Elderly. Geriatrics. Gerontology.
\end{abstract}

\section{REFERÊNCIAS}

ANDREWS, Moya L. Adult and Geriatric Disorders. In: . Manual of Voice Treatment: pediatrics through geriatrics. San Diego: Singular,1995. P. 289-293.

BARBIERI, Natália Alves. Trabalho com Velhos. Algumas Reflexões Iniciais. In: ENCUENTRO LATINOAMERICANO DE PSICANÁLISE, 3., 2002, 
Buenos Aires. Anais.... Buenos Aires: [s.n.], 2002. Disponível em: $<$ http:// www.estadosgerais.org/terceiro_encontro/barbieri-velhos.shtml $>$. Acesso em: 08 abr. 2005

BARROS, Ana Paula Brandão de; CARRARA-ANGELIS, Elizabete. Análise Acústica da Voz. In: DEDIVITIS, Rogério A; BARROS, Ana Paula Brandão de. Métodos de Avaliação e Diagnóstico de Laringe e Voz. São Paulo: Lovise, 2002. P. 201-221.

BEHLAU, Mara Suzana. Presbifonia: envelhecimento vocal inerente à idade. In: RUSSO, Iêda Chaves Pacheco. Intervenção Fonoaudiológica na Terceira Idade. Rio de Janeiro: Revinter, 1999. P. 25-50.

BEHLAU, Mara Suzana, AZEVEDO, Renata; PONTES, Paulo. Voz Normal e Voz Alterada: conceito e classificação das disfonias. São Paulo: Centro de Estudos da Voz, 1999.

BEHLAU, Mara Suzana; MADAZIO, Glaucya; PONTES, Paulo. Avaliação de Voz. São Paulo: Centro de Estudos da Voz, 1999.

BEHLAU, Mara Suzana; PONTES, Paulo. A Avaliação da Voz. In: Avaliação e Tratamento das Disfonias. São Paulo: Lovise, 1995. P. 7998.

BEHLAU, Mara Suzana; PONTES, Paulo. Higiene Vocal: cuidando da voz. Rio de Janeiro: Revinter,1999.

BEHLAU, Mara Suzana; PONTES, Paulo; FEIJÓ, Deborah; MADAZIO Glaucya. Avaliação de Voz. In: BEHLAU, Mara Suzana. (Org.). Voz: o livro do especialista. Rio de Janeiro: Revinter, 2001. V. 1, p. 53-84.

BEHLAU, Mara Suzana; ZIEMER, Roberto. Psicodinâmica Vocal. In: FERREIRA, Léslie P. (Org.) Trabalhando a Voz. São Paulo: Summus, 1988. P. 71-88.

DEDIVITIS, Rogério A.; BARROS, Ana Paula Brandão de. Fisiologia Laríngea. In: Métodos de Avaliação e Diagnóstico de Laringe e Voz. São Paulo: Lovise, 2002. P. 48-50. 
FERREIRA, Ligia Motta; ANUNCIATTO, Nelson F. Envelhecimento Vocal e Neuroplasticidade. In: PINHO, Silvia M. Rebelo. Fundamentos em Fonoaudiologia: tratando os distúrbios da voz. 2. ed. Rio de Janeiro: Guanabara Koogan, 2003. P. 117-37.

GOLDIM, José Roberto; FRANCISCONI, Carlos Fernando; CLOTET, Joaquim. Consentimento Informado e a sua Prática na Assistência e Pesquisa no Brasil. Porto Alegre: Edipucrs, 2000.

MANSUR, Letícia Lessa; RADANOVIC, Márcia. Desenvolvimento da Linguagem no Adulto e no Idoso. In: Neurolingüística: princípios para a prática clínica. São Paulo: Inteligentes, 2004. P. 61-77.

MATSUDO, Sandra M.; MATSUDO, Vitor K. Rodrigues; BARROS NETO, Turíbio Leite. Impacto do Envelhecimento nas Variáveis Antropométricas, Neuromotoras e Metabólicas da Aptidão Física. Revista Brasileira Ciência e Movimento, Brasília, DF, v. 4, p. 21-32, 2000.

MOSES, Paul J. The Voice of Neuroses. New York: Grune \& Stratton, 1954.

POLIDO, Angélica Malena; MARTINS, Maria Ângela dos Santos Ueda Russo; HANAYAMA, Eliana Midori. Percepção do Envelhecimento Vocal na Terceira Idade. Revista CEFAC, São Paulo, v. 7, n. 2, p. 241-51, abr.jun., 2005.

PONTES, Paulo A. L.; VIEIRA, Vanessa P., GONÇALVES, Maria I. R., PONTES, Antônio A. L. Características das Vozes Roucas, Ásperas e Normais: análise acústica espectrográfica comparativa. Revista Brasileira de Otorrinolaringologia, São Paulo, v. 68, n. 2, p. 182-188, 2002.

SATALOFF, Robert Thayer; ROSEN, Deborah Caputo; HAWKSHAW, Mary J.; SIEGEL, Joseph R. The Aging Adult Voice. Journal of Voice, Saint Louis, v. 11, n. 2, p. 156-160, 1997.

SCHNEIDER, Berit; VAN TROTSENBURG, Michael; HANKE, Gunda; BIGENZAHN, Wolfgang; HUBER, Johannes. Voice Impairment and Menopause. Menopause, New York, v. 11, n. 2, p. 151-158, 2004. 
VENITES, Juliana Paula; BERTACHINI, Luciana; RAMOS, Luiz Roberto. Atuação Fonoaudiológica na Presbifonia: a efetividade de uma proposta terapêutica. Fonoaudiologia Brasil, Brasília, DF, v. 4, n. 1, p. 1-8, 2004.

\section{ANEXO}

TABELA 1 - Resultado da avaliação perceptivo-auditiva da voz

\begin{tabular}{|c|c|c|}
\hline Q ualidade de Vida / G rau de A theração & Indivíduos & $\% /$ Total \\
\hline A daptada /N orm al & 2 & 10,53 \\
\hline Rouco-S oprosa ( leve) & 9 & 47,37 \\
\hline Rouco-S oprosa ( m oderada) & 3 & 15,79 \\
\hline Soprosa ( Eve) & 2 & 10,53 \\
\hline Rouco-aspera ( leve) /Soprosa (m oderada) & 1 & 5,26 \\
\hline R ouca Á spera -S oprosa-Trêm ula ( m oderada) & 1 & 5,26 \\
\hline Rouco-S oprosa Áspera ( leve) & 1 & 5,26 \\
\hline Total & 19 & 100,00 \\
\hline
\end{tabular}

TABELA 2 - Resultados da auto-avaliação do impacto vocal na vida particular, social e profissional

\begin{tabular}{|c|c|c|c|c|c|c|}
\hline \multirow{2}{*}{$\begin{array}{l}\text { Tío de vida / } \\
\text { Impacto vocal }\end{array}$} & \multicolumn{2}{|c|}{ ótin o } & \multicolumn{2}{|c|}{ B om } & \multicolumn{2}{|c|}{ R uin } \\
\hline & Indìíluos & $\%$ & Indìíduos & $\%$ & Indìáluos & \% \\
\hline 1. Particuhr & 8 & 42 & 9 & 47 & 2 & 10 \\
\hline 2. Profissional & 10 & 53 & 7 & 36 & 2 & 10 \\
\hline 3.5 ocial & 6 & 31 & 11 & 57 & 2 & 10 \\
\hline
\end{tabular}

